\title{
Surface Deposition and Coalescence and Coacervation Phase Separation Methods: In Vitro Study and Compatibility Analysis of Eudragit RS30D, Eudragit RL30D, and Carbopol-PLA Loaded Metronidazole Microspheres
}

\author{
Irin Dewan, ${ }^{1}$ Md. Maynul Islam,, Maksud Al-Hasan, ${ }^{1}$ Joydeb Nath, \\ Sefat Sultana, ${ }^{1}$ and Md. Sohel Rana ${ }^{2}$ \\ ${ }^{1}$ Department of Pharmacy, University of Asia Pacific, Dhanmondi, Dhaka 1209, Bangladesh \\ ${ }^{2}$ Department of Pharmacy, Jahangirnagar University, Savar, Dhaka 1342, Bangladesh
}

Correspondence should be addressed to Irin Dewan; irin_d@yahoo.com

Received 12 July 2015; Revised 14 October 2015; Accepted 25 October 2015

Academic Editor: Anthony A. Attama

Copyright (c) 2015 Irin Dewan et al. This is an open access article distributed under the Creative Commons Attribution License, which permits unrestricted use, distribution, and reproduction in any medium, provided the original work is properly cited.

Metronidazole (MTZ) has extremely broad spectrum of protozoal and antimicrobial activity and is clinically effective in trichomoniasis, amoebic colitis, and giardiasis. This study was performed to formulate and evaluate the MTZ loaded microspheres by coacervation phase separation and surface deposition and coalescence methods using different polymers like Gelatin, Carbopol 934P, Polylactic Acid (PLA), Eudragit RS30D, and Eudragit RL30D to acquire sustained release of drug. In vitro dissolution studies were carried out in phosphate buffer ( $\mathrm{pH}$ 7.4) for 8 hours according to USP paddle method. The maximum and minimum release of MTZ from microspheres observed were $84.81 \%$ and $76.6 \%$ for coacervation and $95.07 \%$ and $80.07 \%$ for surface deposition method, respectively, after 8 hours. Release kinetics was studied in different mathematical release models. The SEM and FTIR studies confirm good spheres and smooth surface as well as interaction between drug and polymers. Though release kinetic is uncertain, the best fit was obtained with the Korsmeyer kinetic model with release exponent $(n)$ lying between 0.45 and 0.89 . In vitro studies showed that MTZ microspheres with different polymers might be a good candidate as sustained drug delivery system to treat bacterial infections.

\section{Introduction}

In the early 1950s Barrett K. Green developed the microencapsulation that used the process of coacervation. The first pharmaceutical product consisting of microcapsules was a controlled release aspirin product. The oral route is considered as the most promising route of drug delivery. Conventional drug delivery system achieves and maintains the drug concentration within the therapeutically effective range needed for treatment, only when taken several times a day. This results in a significant fluctuation in drug levels. A well-defined controlled drug delivery system can overcome some of the problems of conventional therapy and enhance the therapeutic efficacy of a given drug [1]. For colonic drug delivery, many physiological barriers must be overcome, the major one being absorption or degradation of the active drugs in the upper part of the GIT. Most of the peptide and protein drugs are unstable in the stomach and upper part of the intestine. Colon specific drug delivery protects peptide drug from releasing. After bioavailability, there should be no space before the period. To achieve successful colonic delivery, a drug needs to be protected from absorption and/or degradation in the environment of the upper GIT [2].

A variety of controlled release systems such as coated pellets, matrix tablets, osmotically controlled release systems, microcapsules, microspheres, nanoparticles, implants, and infusion devices have been designed for various routes of drug administration. Controlled release formulations in tablet form are many, but over the years the microsphere formulations have immense popularity owing to their superiority over the former in several respects [3]. A multipleunit dosage form has more homogenous individual plasma 
TABLE 1: Formulation and drug loading efficiency of metronidazole microspheres prepared by coacervation phase separation method and surface deposition and coalescence.

\begin{tabular}{|c|c|c|c|c|c|c|c|c|}
\hline \multirow{2}{*}{$\begin{array}{l}\text { Formulation } \\
\text { code }\end{array}$} & \multirow{2}{*}{ Drug (gm) } & \multicolumn{3}{|c|}{ Polymers (gm) } & \multirow{2}{*}{ Formaldehyde $(\mathrm{mL})$} & \multirow{2}{*}{$\mathrm{AL}(\%)$} & \multirow{2}{*}{$\operatorname{DEE}(\%)$} & \multirow{2}{*}{ Methods } \\
\hline & & Gela & Car & PLA & & & & \\
\hline $\mathrm{F} 25$ & 1 & 1 & 0 & 0 & 0.5 & 14.91 & 74.58 & \multirow{3}{*}{ Coacervation phase separation } \\
\hline F26 & 1 & 1 & 0.5 & 0 & 0.5 & 16.48 & 82.04 & \\
\hline F27 & 1 & 1 & 0 & 0.5 & 0.5 & 16.11 & 80.55 & \\
\hline $\mathrm{F} 28$ & 10 & EuRS30D: 6.6 & - & - & - & 14.72 & 73.62 & \multirow{2}{*}{ Surface deposition and coalescence } \\
\hline F29 & 10 & EuRL30D: 6.6 & - & - & - & 14.48 & 72.04 & \\
\hline
\end{tabular}

Gela: Gelatin, Car: Carbopol, PLA: Polylactic Acid, EuRS30D: Eudragit RS30 dispersion, EuRL30D: Eudragit RL30 dispersion, DEE: drug entrapment efficiency, AL: actual loading, mL: milliliter, and gm: gram.

profiles, shorter lag time, and lower variability as compared to single-unit formulations. The uniform distribution of these multiple unit dosage forms along the GIT could result in more reproducible drug absorption and reduced risk of local irritations than the use of single-unit dosage forms. Risks such as spontaneous drug release from a single-unit tablet due to damaged coating or its attachment in the stomach or intestine causing an irritation of the gastric or intestinal mucosa are reduced by the use of multiunit forms. Thus, it results in a decrease in drug dose and side effects [4].

Due to the smallness of the particles, drugs can be widely distributed throughout the GI tract, hence improving the drug absorption. The microencapsulation technique offers a variety of opportunities such as protection and masking, reduced dissolution rate, facilitation of handling, and spatial targeting of the active ingredient. This approach facilitates accurate delivery of small quantities of potent drugs, reduced drug concentrations at sites other than the target organ or tissue, and protection of labile compounds before and after administration and prior to appearance at the site of action. In the future by combining various other approaches, microencapsulation technique will find the vital place in novel drug delivery system [5].

Metronidazole, a nitroimidazole, has extremely broad spectrum of antiprotozoal and antimicrobial activities, with high activity against anaerobic bacteria. Metronidazole is sparingly soluble in water and alcohol and slightly soluble in ether, chloroform, acetone, and methanol. Half-life of metronidazole is about 6 to 7 hours [6]. The sustained release MTZ microspheres can be prepared by different microencapsulation technique. Microencapsulation is the process in which small droplets or particles of liquid or solid material are surrounded or coated by a continuous film of polymeric materials. The purpose of microencapsulation is to improve the solubility, increase bioavailability, and delay the release of drug [7]. Coacervation phase separation process may be used to microencapsulate a variety of liquids, solids, solutions, and dispersions of solids in liquids. The polymers used to coat the materials should be soluble in water or any other solvent used. Water-soluble core materials are microencapsulated in organic solvents, whereas water-insoluble materials are microencapsulated in water [8].

Methacrylate copolymers (Eudragits) have recently received increased attention for modified dosage forms because of their inertness, solubility in relatively nontoxic solvents, and availability of resins with different properties. Eudragit RS30D and Eudragit RL30D are a copolymer of ethyl acrylate, methyl methacrylate, and a low content of methacrylic acid ester with quaternary ammonium groups. The ammonium groups are present as salts and make the polymers permeable [9]. Carbopol is a polymer consisting of acrylic acid cross-linked with either polyalkenyl ether or divinyl glycol. It readily absorbs water, gets hydrated, and swells. In addition to its hydrophilic nature, crosslinked structure, and insolubility in water, Carbopol is an anionic polymer and, therefore, a potential candidate for use in controlled release drug delivery [10]. Polymers such as polylactide (PLA) and poly(lactide-co-glycolide) (PLGA) have been successfully used to prepare microand nanoparticulate drug delivery systems as well as other medical devices, like sutures and implants. Pharmaceutical products based on PLA and PLGA microparticles loaded with hormones, antitumor drugs, and antibiotics are already available in the market in Europe and the US [11].

The aim of the present investigation was to formulate and in vitro evaluate colon targeted microspheres of metronidazole using a combination of time controlled and $\mathrm{pH}$ independent polymethacrylate polymers that offer protection to the drug until it leaves the stomach and major drug release in small intestine is avoided by providing $\mathrm{pH}$ independent coating of Eudragits RS and RL.

\section{Materials and Methods}

2.1. Materials. The materials used were metronidazole as a donation sample from SQUARE Pharmaceuticals Ltd., Bangladesh, Carbopol 934P (Colorcon Asia Pvt. Limited, India), Polylactic Acid (PLA) (Merck, Germany), Eudragit RS30D (Evonik, Germany), Eudragit RL30D (Evonik, Germany), light liquid paraffin, petroleum ether (Merck, Germany), sodium hydroxide (Merck, Germany), potassium dihydrogen phosphate (Merck, Germany), and distilled water.

\subsection{Methods}

2.2.1. Preparation of Metronidazole Microspheres by Coacervation Phase Separation Method. According to Table 1 GelatinCarbopol/PLA mixture was dissolved in ten $\mathrm{mL}$ of water 


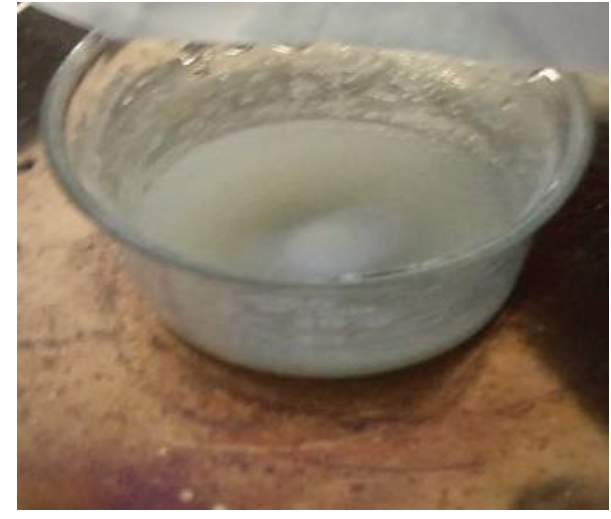

(a) Gelatin-Carbopol/PLA mixture was prepared

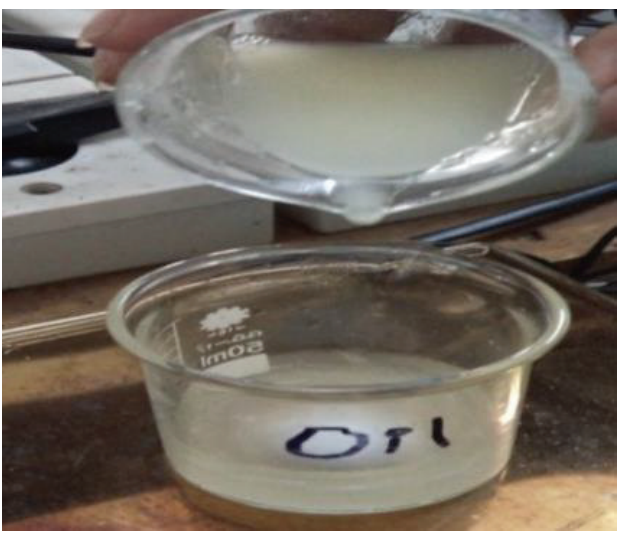

(c) Drug-polymer dispersion was added dropwise to sunflower oil

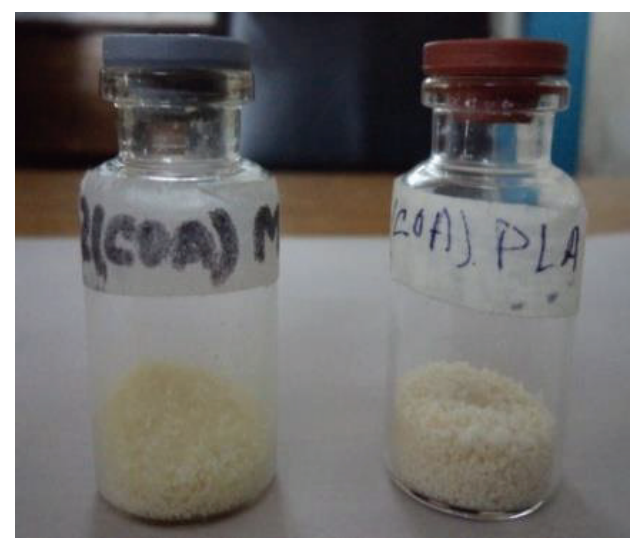

(e) Finally microspheres are stored in airtight container

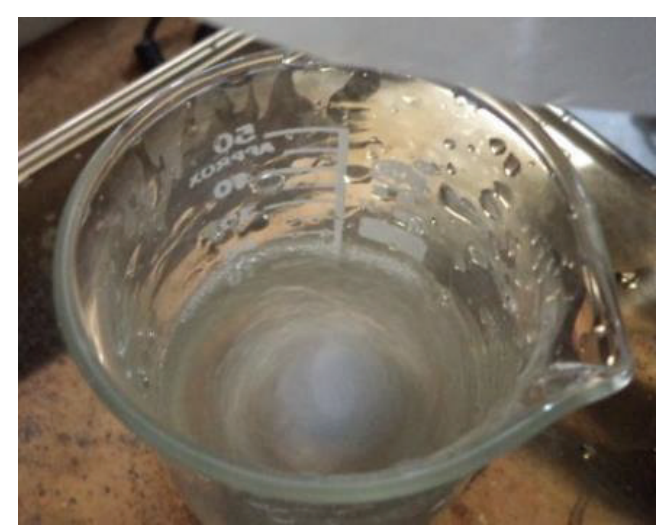

(b) Drug-polymer dispersion was prepared

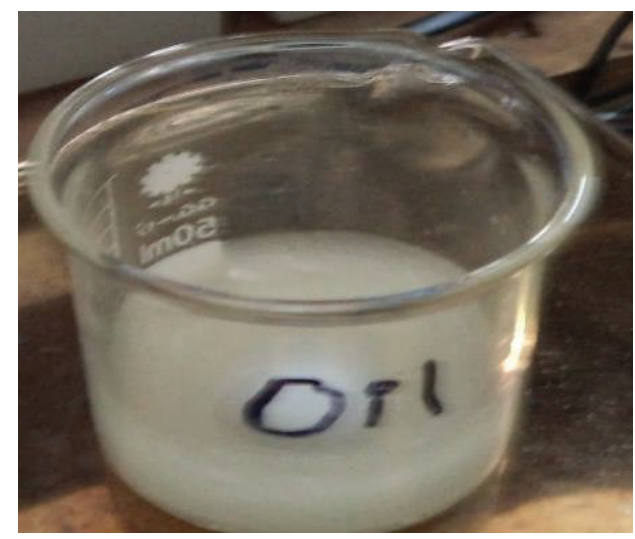

(d) Formaldehyde was added and stored in refrigerator for 24 hours for rigidization

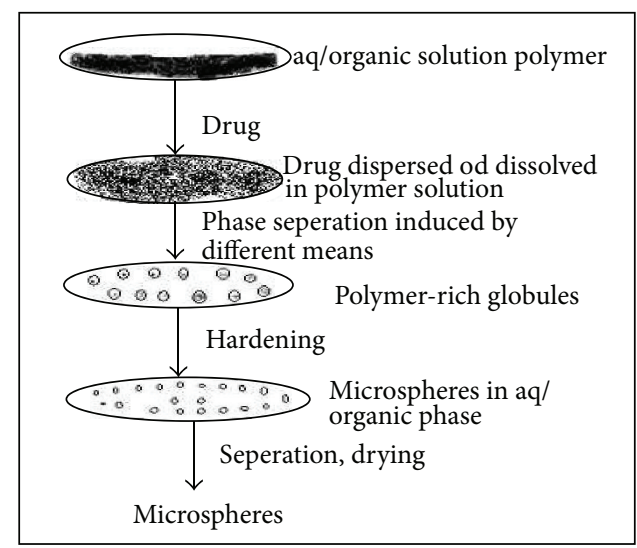

(f) Flow diagram of coacervation method

FIGURE 1: It schematically presents the steps of microspheres prepared by coacervation phase separation method.

which was previously heated to $50^{\circ} \mathrm{C}$. To this metronidazole was added and stirred approximately at $300 \mathrm{rpm}$ with the help of magnetic stirrer for 15 minutes to get a stable dispersion. The dispersion was poured dropwise into the ten $\mathrm{mL}$ of sunflower oil which was also previously heated to $50^{\circ} \mathrm{C}$ on a water bath. The mixture was stirred with a help of magnetic stirrer for $2 \mathrm{hrs}$ at $300 \mathrm{rpm}$ at room temperature. At the end of two hrs, cross-linking agent formaldehyde $0.5 \mathrm{~mL}$ was added to the dispersion medium with continuous stirring for next 30 minutes. After that, the final dispersion was kept in refrigerator for $24 \mathrm{hrs}$ to make sure of the rigidization of microspheres shown in Figure 1.

\subsubsection{Preparation of Metronidazole Microspheres Based on Surface Deposition and Coalescence Method}

Step 1 (preparation of granules using Eudragit RL/RS30D by wet granulation method). For the preparation of granules at 


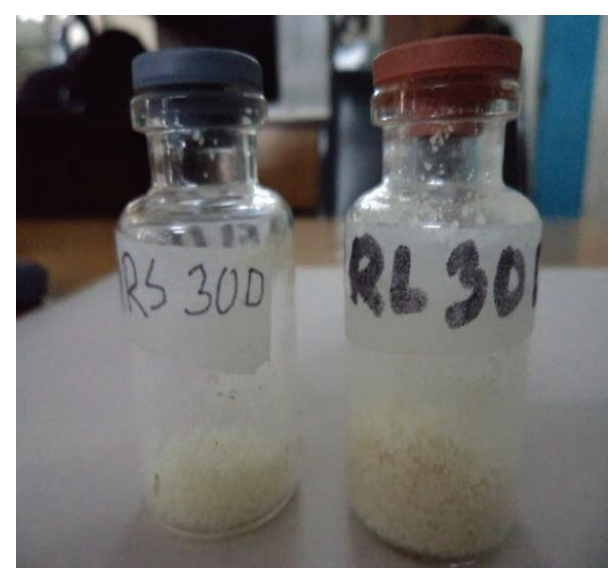

FIGURE 2: Formulation of metronidazole microspheres based on surface deposition and coalescence method.

first ten grams $(10 \mathrm{gm})$ of drug was placed in a beaker and $6.6 \mathrm{gm}$ of Eudragit RL/RS30D was added to it in aliquots with proper mixing. The wet mass was air-dried and passed twice through 30-mesh sieve and kept in an oven at $40^{\circ} \mathrm{C}$ for 2 hours. After drying the granules were sieved through 710-, 500-, and 250-micron-size sieves and collected in different size ranges.

Step 2 (preparation of microspheres using emulsion solvent evaporation method). According to Table 1 about $300 \mathrm{gm}$ of light liquid paraffin oil and $1 \mathrm{gm}$ of span 20 were mixed in a beaker with continuous stirring at $2000 \mathrm{rpm}$ for few minutes. The whole mixture was divided into two parts (part A: $200 \mathrm{gm}$ and part B: $100 \mathrm{gm})$.

Part A was taken in a beaker and an emulsion was made with 18 gm Eudragit RL/RS30D. Part B was taken in another beaker and placed onto a hot plate. The temperature was raised to $90^{\circ} \mathrm{C}$. About $4 \mathrm{gm}$ of $250-500$-micron-sized metronidazole granules was added to this part of light liquid paraffin with continuous stirring. Part A containing Eudragit RL/RS30D emulsion was added in different installment. Few-minute time was allowed between each addition to settle down the Eudragit RL/RS30D over the granules. The temperature was maintained at 85 to $90^{\circ} \mathrm{C}$ for 90 minutes. After cooling to room temperature the microspheres were filtered and washed three times with petroleum ether and those were kept in dry air. Finally the microspheres were dried at $40^{\circ} \mathrm{C}$ for 12 hours shown in Figure 2.

2.3. Assay Methods of Prepared Microspheres. Approximately $20 \mathrm{mg}$ of microspheres was taken in a $100 \mathrm{~mL}$ volumetric flask and dissolved in up to phosphate buffer ( $\mathrm{pH}$ 7.4) and thoroughly shaken in ultrasonic bath at $37^{\circ} \mathrm{C}$ for 30 minutes. Then the solution was filtered through Whatman filter paper and analyzed spectrophotometrically at $277 \mathrm{~nm}$ for the drug concentration. The drug loading was calculated by using the following equation:

$$
\begin{aligned}
\% \text { Drug Loading }= & \frac{\text { Actual Drug Loading }}{\text { Microspheres to Be Taken }} \\
& \times 100 \% .
\end{aligned}
$$

The drug entrapment efficiency was calculated by using the following equation:

$$
\begin{aligned}
& \text { \%Drug Entrapment Efficiency } \\
& \qquad=\frac{\text { Actual Drug Content }}{\text { Theoretical Drug Content }} \times 100 \%
\end{aligned}
$$

2.4. In Vitro Release Study of Metronidazole Microspheres. In vitro dissolution study was performed in a paddle type dissolution apparatus. Nine hundred milliliters $(900 \mathrm{~mL})$ of phosphate buffer ( $\mathrm{pH}$ 7.4) was used as dissolution media, paddle speed was $100 \mathrm{rpm}$, and temperature was maintained fixed at $37^{\circ} \mathrm{C}$. Approximately $20 \mathrm{mg}$ of microspheres from each batch was transferred into each dissolution basket. The dissolution process was carried out for 8 hours and $10 \mathrm{~mL}$ dissolution sample was withdrawn at predetermined different time intervals and replaced with the same volume of test medium to maintain sink conditions. Dissolution samples were withdrawn with the help of $10 \mathrm{~mL}$ syringe and kept in test tubes. The withdrawn samples were diluted, where necessary, filtered through $0.45 \mu$ membrane filter, and analyzed in UV-VIS spectrophotometer at a wavelength of $277 \mathrm{~nm}$.

2.5. Kinetic Analysis of Dissolution Data. To study the mechanism of drug release from the microspheres, the release data were fitted to zero order, first order, and Higuchi equation [12]. Moreover, for better characterization of the drug release mechanisms, the Korsmeyer-Peppas [13] semiempirical model was applied:

$$
\frac{Q_{t}}{Q_{e}}=k_{\mathrm{KP}} t^{n},
$$

where $Q_{t} / Q_{e}$ is the fraction of the drug released at time $t, k_{\mathrm{KP}}$ is a constant corresponding to the structural and geometric characteristics of the device, and $n$ is the release exponent which is indicative of the mechanism of the drug release. To clarify the release exponent batches of microspheres, the $\log$ value of percentage drug dissolved was plotted against log time for each batch according to the above equation. A value of $n=0.45$ indicates Fickian (case I) release; $>0.45$ but $<0.89$ indicates non-Fickian (anomalous) release; and $>0.89$ indicates super case II type of release. Case II generally refers to the erosion of the polymeric chain and anomalous transport (non-Fickian) refers to a combination of both diffusion and erosion controlled drug release [14]. Electron Microscope (SEM). Surface nature of microspheres 
TABLE 2: Correlation coefficient $\left(R^{2}\right)$ of different formulations of metronidazole microspheres using different polymers by coacervation and coalescence methods, respectively.

\begin{tabular}{lccccc}
\hline \multirow{2}{*}{ Formulation code } & Zero order & First order & Higuchi model & \multicolumn{2}{c}{ Korsmeyer model } \\
& $R^{2}$ & $R^{2}$ & $R^{2}$ & $R^{2}$ & 0.8405 \\
F25 & 0.9721 & 0.9773 & 0.9682 & 0.9795 & 15.71 \\
F26 & 0.9716 & 0.9805 & 0.9659 & 0.9935 & 14.32 \\
F27 & 0.9483 & 0.9756 & 0.9573 & 0.9891 & 13.88 \\
F28 & 0.9494 & 0.9633 & 0.9809 & 0.984 & 22.70 \\
F29 & 0.9855 & 0.9811 & 0.9642 & 0.9971 & 0.879 \\
\hline
\end{tabular}

was examined with the help of Scanning Electron Microscope (JEOL, JSM-6490 LA, Japan). The microspheres were dried completely before examination. SEM was done at different magnifications of $20.0 \mathrm{kv} \times 75,20.0 \mathrm{kv} \times 90,20.0 \mathrm{kv} \times 95$, $20.0 \mathrm{kv} \times 140,20.0 \mathrm{kv} \times 300,20.0 \mathrm{kv} \times 600$, and $20.0 \mathrm{kv} \times 1000$.

\subsection{Fourier Transform Infrared (FTIR) Spectroscopy Studies.} The FTIR technique is to measure the absorption of various infrared radiations by the target material and to produce an IR spectrum that can be used to identify functional groups and molecular structure in the sample shown in Figure 7. FTIR spectra of pure MTZ and formulated microspheres were recorded by using FTIR 8400S (SHIMADZU, Japan). Appropriate quantities of $\mathrm{KBr}$ and microspheres (in the ratio $100: 2$ ) were mixed by grinding in an agate mortar. Disk was made with about $100 \mathrm{mg}$ mixture under hydraulic pressure of $600 \mathrm{~kg}$. Then the FTIR spectra were recorded between 4000 and $400 \mathrm{~cm}^{-1}$. The resolution was $2 \mathrm{~cm}^{-1}$.

\section{Results and Discussions}

3.1. Actual Drug Loaded and Drug Entrapment Efficiency (DEE) of Prepared Microspheres. Drug loading and the drug entrapment efficiency (DEE) of the prepared microspheres were carried out and the graphical presentation is given in Figure 3. The actual drug loaded and the drug entrapment efficiency were found to be in the range of $14.48 \%$ to $16.48 \%$ and $72.04 \%$ to $82.04 \%$, respectively.

\subsection{Dissolution Study of Metronidazole Microspheres Prepared} by Coacervation Phase and Surface Deposition and Coalescence Methods. To find out the mechanism of drug release, the controlled release MTZ microspheres were treated in different mathematical models like zero order (cumulative percentage of drug release versus time), first order (log percentage of drug remaining versus time), Higuchi model (cumulative percentage of drug release versus square root of time), and Korsmeyer model (log cumulative percentage of drug release versus log time). The release data was plotted. From the linear portions of the curve slope correlation coefficients $\left(R^{2}\right)$ were calculated. With the Korsmeyer plot, linearity was noted highest in all formulations using all data points. The data yielded apparently straight line with Korsmeyer plot $\left(R^{2}>0.99\right)$ while a bit with zero order, first order kinetics and Higuchi plot. It is observed that

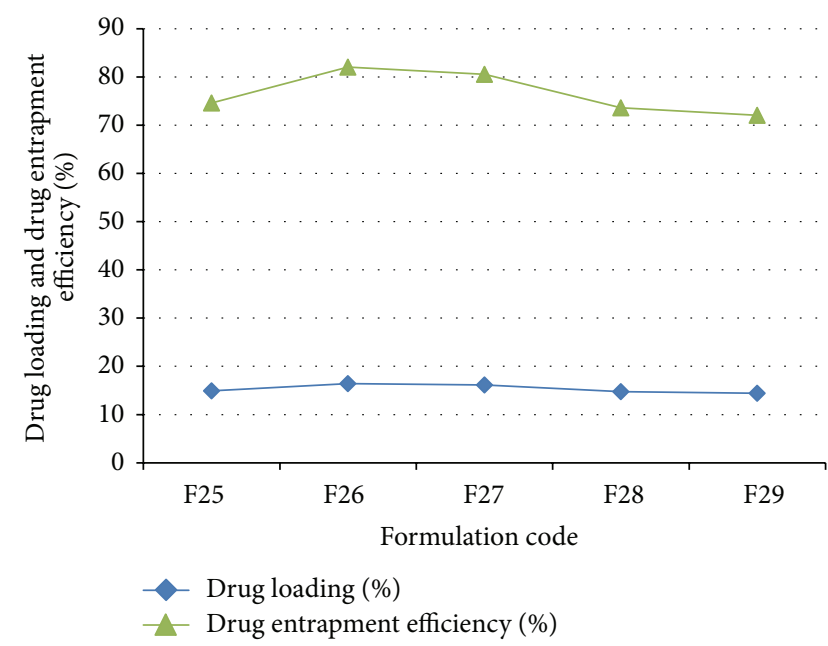

FIgURE 3: Comparative percent release study of actual drug loading and drug entrapment efficiency of different formulations.

drug released from sustained release microsphere followed Korsmeyer release log cumulative percentage of drug release versus log time. The mechanism of drug release was calculated according to Peppas equation. The calculated " $n$ " values along with the correlation coefficients $\left(R^{2}\right)$ have been shown in Table 2 . The values of $n$ depend upon the polymer concentration. The calculated " $n$ " values suggest that the mechanism of drug release followed non-Fickian transport.

\subsection{Effect of Polymers (Gelatin, Carbopol, and Polylactic Acid)} and (Eudragit RL30D and Eudragit RS30D) on the Release of Metronidazole Microspheres Prepared by Coacervation Phase Separation and Surface Deposition and Coalescence Methods, Respectively. Metronidazole microspheres were prepared by polymeric concentration variation to study the effect of combination polymer on the release of drug from microspheres.

Polymers such as Gelatin, Carbopol, and Polylactic Acid (PLA) in different concentrations were used in formulations F25 to F27. The initial burst release of formulations F25, F26, and F27 was about $13.68 \%, 12.73 \%$, and $12.26 \%$, respectively, after 1 hour (Figure 4 ). After the end of 8 hours of dissolution, the release of microspheres from F25, F26, and F27 was $84.81 \%, 78.49 \%$, and $76.60 \%$, respectively. The addition of Carbopol along with Gelatin in formulation F26 retards the 


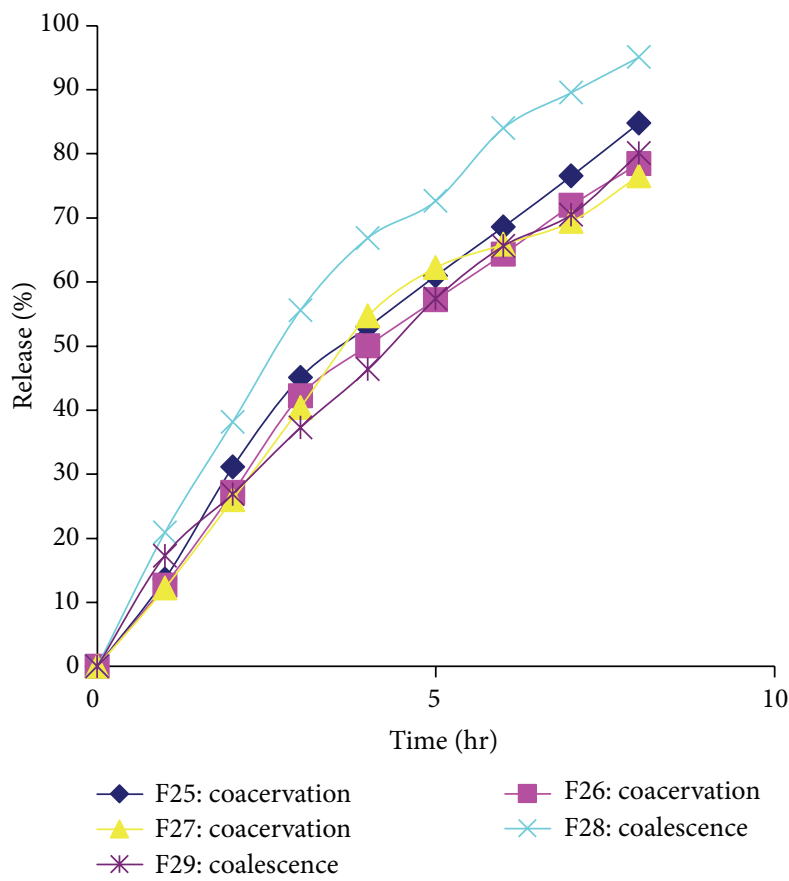

(a)

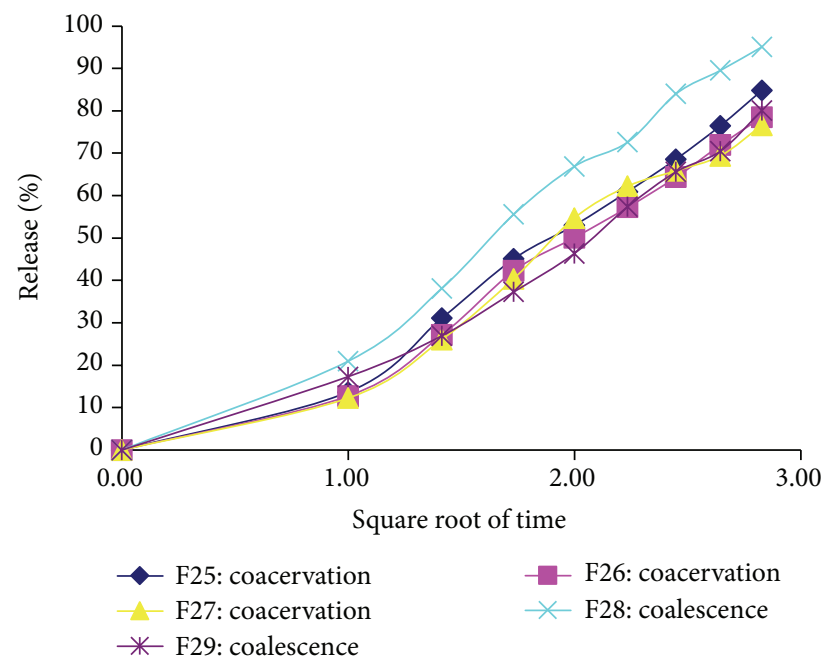

(c)

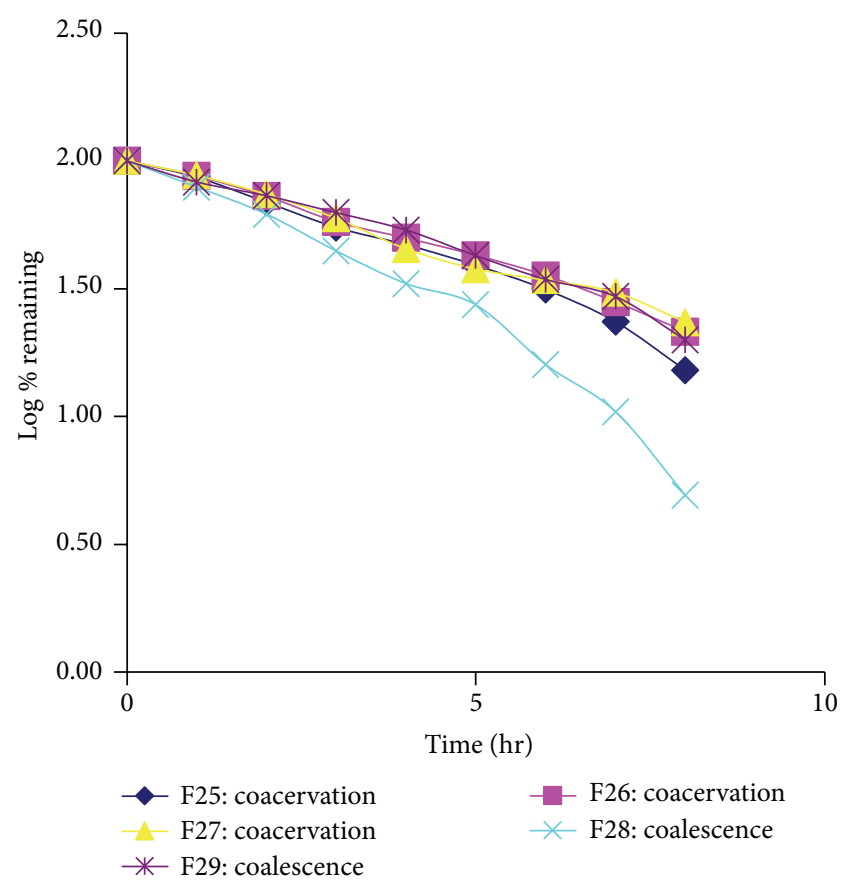

(b)

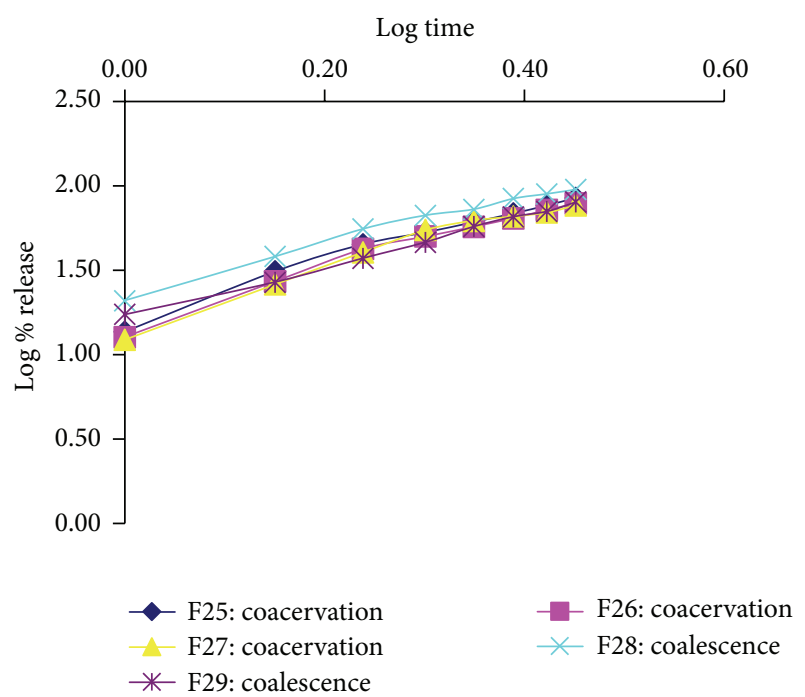

(d)

FIGURE 4: Release of metronidazole from formulations F25 to F27 and formulations F28 to F29 by coacervation phase separation and surface deposition and coalescence method, respectively. (a) Zero order, (b) first order, and (c) Higuchi and (d) Korsmeyer models.

rate of drug release. The addition of PLA along with the same concentration of Gelatin in formulation F27 retards the rate of drug release significantly. Besides formulations F28 and F29 were prepared using the polymers of Eudragit RL30D and Eudragit RS30D in different concentrations. The initial burst release of formulations F28 and F29 was about 20.94\% and $17.31 \%$, respectively, after 1 hour (Figure 4 ). After the end of 8 hours of dissolution, the release of microspheres from F28 and F29 was $95.07 \%$ and $80.07 \%$, respectively. The release of drug is significantly higher for formulation F28 than formulation F29. Eudragit RS30D retards the release of drug more than Eudragit RL30D.

All the formulations were best fitted with Korsmeyer model as shown in Table 2. The release exponent $n>0.45$ and $n<0.89$ for all the formulations showed non-Fickian (case II) anomalous release of drug which refers to a combination of both diffusion and erosion controlled drug release.

3.4. Effect of Polymers Concentration on the Surface Morphology of Metronidazole Microspheres Prepared by Coacervation 


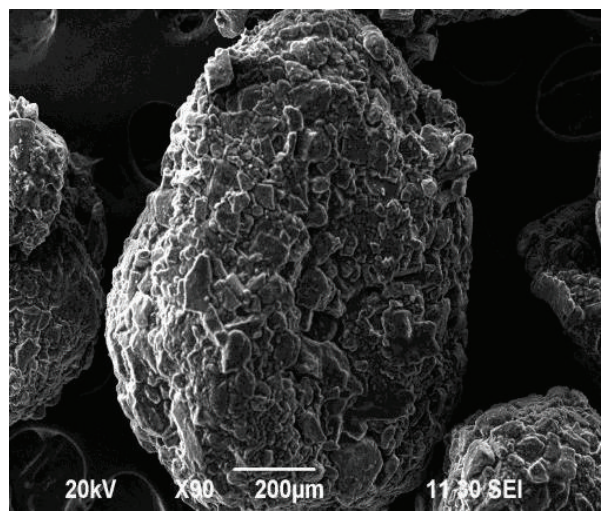

(a)

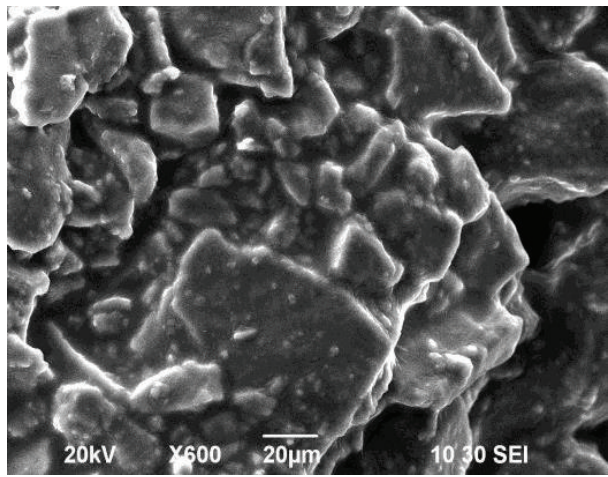

(c)

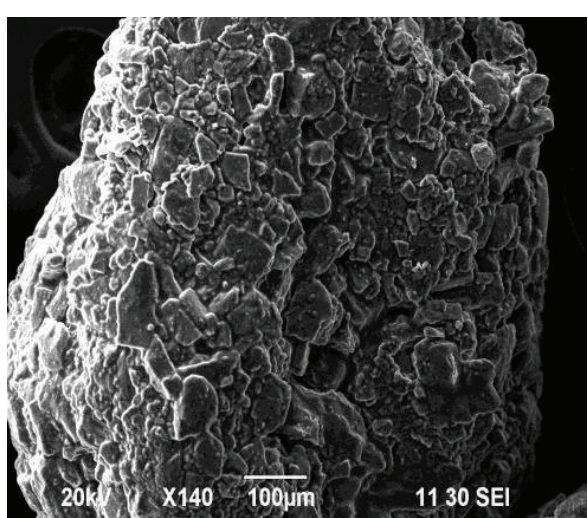

(b)

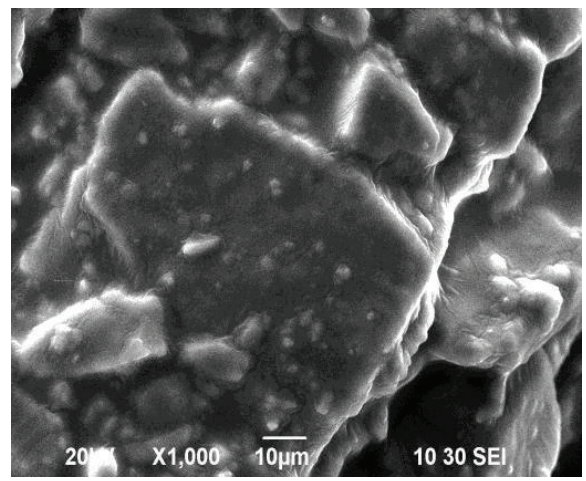

(d)

FIGURE 5: SEM studies of metronidazole microspheres of formulation F25 prepared by coacervation phase separation method with different magnification: (a) at $\times 90$ SEI, (b) at $\times 140$ SEI, (c) at $\times 600$ SEI, and (d) at $\times 1000$ SEI.

Phase Separation Method. SEM study has shown that microspheres of formulation F25 were spherical and aggregated. The surface of the drug loaded microspheres manifested the presence of drug particles and rough surface, clearly visible from outside at high magnification in Figure 5.

3.5. Effect of Polymers Concentration on the Surface Morphology of Metronidazole Microspheres Prepared by Surface Deposition and Coalescence Method. Formulation F28 has contained the polymer Eudragit RL30D. The microspheres were found in spherical shape and surface was smooth as shown in Figure 6. The magnification of microcapsules showed that the polymers surround the drug.

3.6. Drug-Polymer Compatibility Study by Fourier Transform Infrared (FTIR) Spectroscopy. FTIR spectra of pure MTZ and its combination with polymers were shown in Figure 7. An FTIR spectrum of pure metronidazole showed the peaks such as $\mathrm{O}-\mathrm{H}$ stretch $\left(3211.8 \mathrm{~cm}^{-1}\right), \mathrm{C}=\mathrm{CH}, \mathrm{C}-\mathrm{H}$ stretch $\left(3099.61 \mathrm{~cm}^{-1}\right), \mathrm{NO} 2, \mathrm{~N}-\mathrm{O}$ stretch $\left(1533.41 \mathrm{~cm}^{-1}\right), \mathrm{C}-$ $\mathrm{OH}, \mathrm{C}-\mathrm{O}$ bend $\left(1074.35 \mathrm{~cm}^{-1}\right)$, and $\mathrm{C}-\mathrm{NO} 2, \mathrm{C}-\mathrm{N}$ stretch $\left(825.53 \mathrm{~cm}^{-1}\right)$ that are shown in Table 3 . These peaks can be considered as characteristic peaks of drug and were not affected and prominently observed in IR spectra of pure drug along with polymers as shown in Figures 7 (b) and 7 (c), indicating no interaction between drug and polymers. These peaks indicated that MTZ is entrapped here. As various polymers were used in those formulations in different amounts, the IR spectra were different from the active one.

\section{Conclusion}

The present study was conducted to design metronidazole sustained release microspheres by coacervation phase separation and surface deposition and coalescence method. Different polymers of different concentrations were used in the preparations to observe the surface of microspheres and thus the release characteristics of drug. As the concentration of polymer increases, the release rate decreases gradually and the release studies showed that highest concentration of polymer gave the best sustained effect. All the formulations were best fitted with Korsmeyer model. The release exponent $n<0.89$ for all the formulations showed nonFickian (case II) anomalous release of drug which refers to a combination of both diffusion and erosion controlled drug release.

Further experiment should be performed to establish more sustained effect. This approach facilitates accurate delivery of small quantities of potent drugs, reduced drug 
TABLE 3: Functional group detection using wave number.

\begin{tabular}{lccc}
\hline Functional group & \multicolumn{2}{c}{ Wave number in 1/cm } \\
C-H alkenes & Range & Formulation F26 & Formulation F28 \\
C-CI & $700-610$ & $677.01-630.72$ & 723.31 \\
C-N (bend) & $735-702$ & $98.94-630.72$ \\
C-O (bend) & $1000-750$ & 917.52 & $989.48-825.53$ \\
C-N & $1300-1000$ & $1294.24-1002.98$ & $1290.38-1033.85$ \\
N=O & $1350-1000$ & $1340.53-1002.98$ & $1340.53-1033.85$ \\
-CHO & $1550-1350$ & $1467.83-1357.89$ & $1533.41-1354.03$ \\
Aliphatic & $1720-1740$ & & $3099.61-2852$ \\
C-H (stretch) & $3100-2850$ & $3099.61-2854.65$ & 3213.41 \\
O-H & $3400-3200$ & $3375.43-3213.41$ & \\
\hline
\end{tabular}

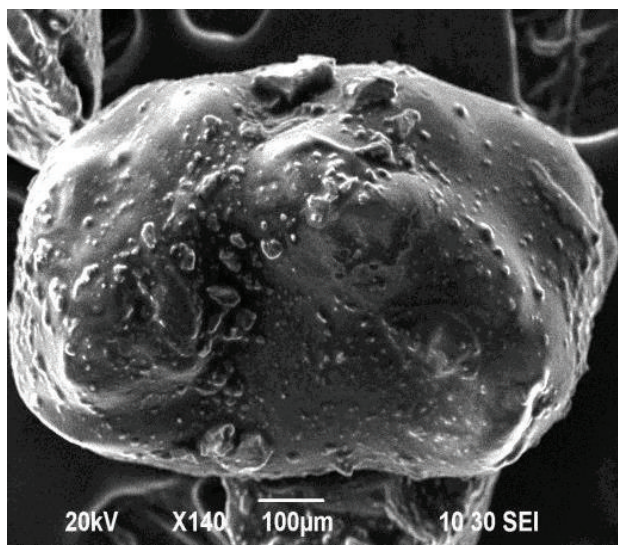

(a)

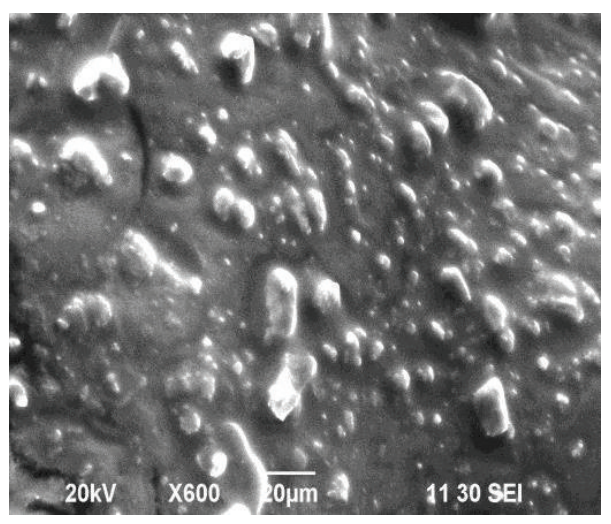

(c)

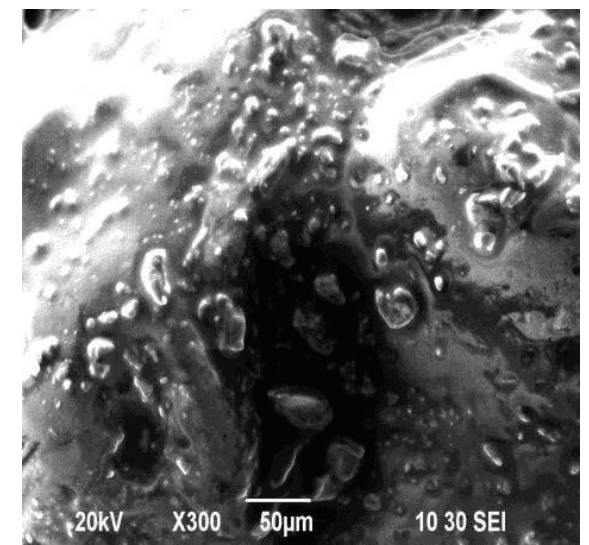

(b)

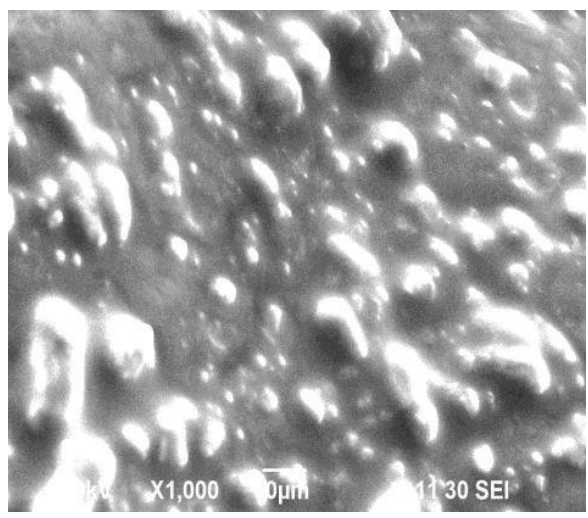

(d)

FIGURE 6: SEM studies of metronidazole microspheres of formulation F28 prepared by surface deposition and coalescence method with different magnification: (a) at $\times 140$ SEI, (b) at $\times 300$ SEI, (c) at $\times 600$ SEI, and (d) at $\times 1000$ SEI.

concentrations at sites other than the target organ or tissue, and protection of labile compounds before and after administration and prior to appearance at the site of action. In vitro studies showed that MTZ microspheres with different polymers might be a good candidate as sustained drug delivery system to treat bacterial infections. It can be concluded that polymers lower the release rate of the drug and may prolong the activity and overall release kinetics.

\section{Conflict of Interests}

The authors affirm that there is no conflict of interests concerning the publication of this paper. 


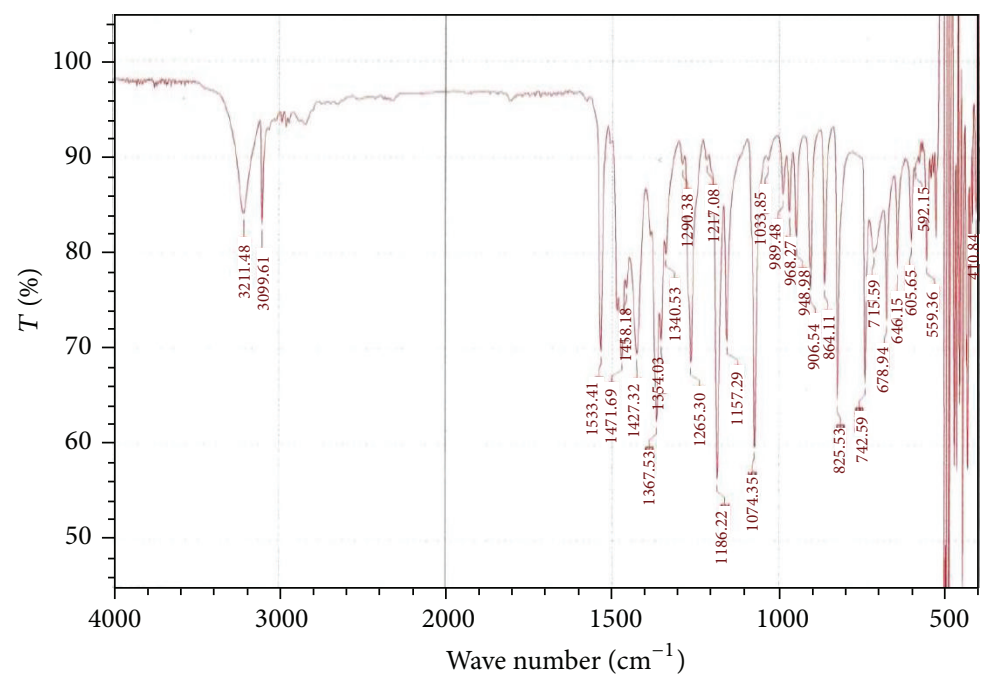

(a)

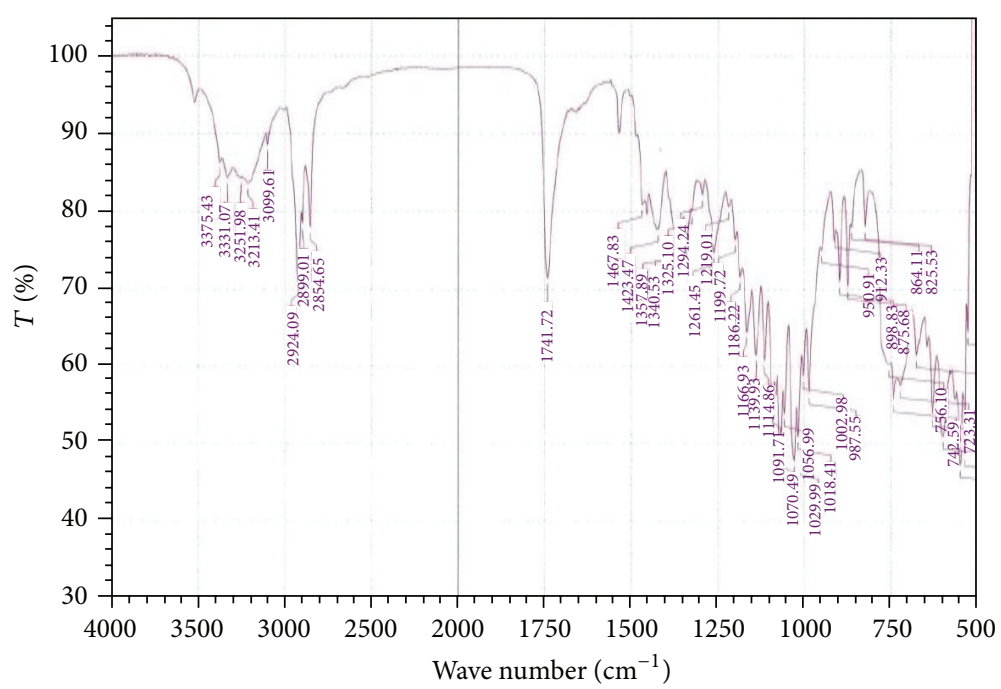

(b)

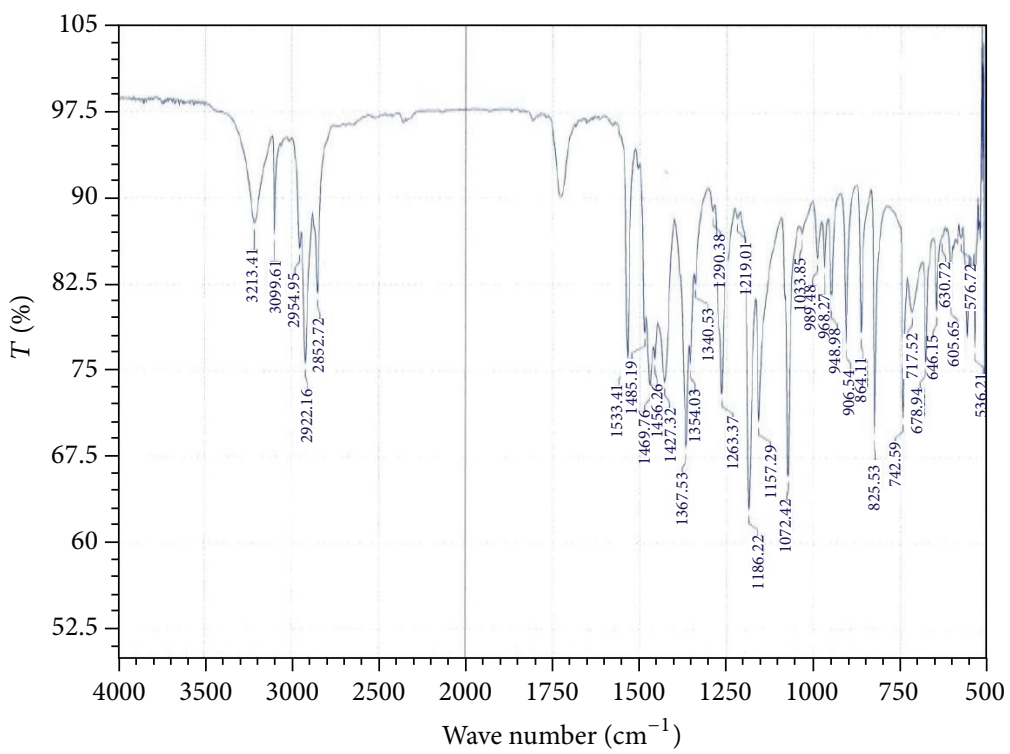

(c)

FIGURE 7: FTIR spectrum of (a) pure drug metronidazole, (b) formulation F26, (c) formulation F28, Eudragit RS30D, and Eudragit RL30D, respectively. \% T: \% transmittance or absorbance. 


\section{Acknowledgments}

The authors are grateful to the SQUARE Pharmaceuticals Ltd., Bangladesh, for giving active ingredients as a gift and the University of Asia Pacific for providing facilities to carry out this research work.

\section{References}

[1] P. Srivastava and S. Visht, "Application and advancement of microsphere as controlled delivery system: a review," International Journal of Pharmacy \& Life Sciences, vol. 4, no. 4, pp. 2583-2594, 2013

[2] B. Satish, L. M. Bikash, and Z. Tomal, "Design and evaluation of sustained release microspheres," Indian Journal of Clinical Practice, vol. 22, pp. 125-128, 2012.

[3] M. K. Chourasia and S. K. Jain, "Pharmaceutical approaches to colon targeted drug delivery systems," Journal of Pharmacy and Pharmaceutical Sciences, vol. 6, no. 1, pp. 33-66, 2003.

[4] M. U. Uhumwangho and R. S. Okor, "Estimation of the release profiles of multi-unit dose tablets of theophylline from the release profiles of their components," Tropical Journal of Pharmaceutical Research, vol. 7, no. 2, pp. 981-986, 2008.

[5] S. P. Vyas and R. K. Khar, Targeted and Controlled Drug Delivery: Novel Carrier System, CBS Publishers and Distributors, New Delhi, India, 2002.

[6] G. Tiwari, "Biodegradable microspheres for controlled delivery of metronidazole in the treatment of periodontal diseases: formulation consideration," International Journal of Pharma and Bio Sciences, vol. 1, no. 2, article 82, 13 pages, 2010.

[7] I. Dewan, S. Miah, S. M. A. Islam, and S. Rana, "Design, characterization and in-vitro evaluation of different cellulosic acrylic and methacrylic polymers loaded aceclofenac microspheres," Pakistan Journal of Pharmaceutical Sciences, vol. 27, no. 5, pp. 1241-1248, 2014.

[8] M. A. Bayomi, S. A. Al-Suwayeh, A. M. El-Helw, and A. F. Mesnad, "Preparation of casein-chitosan microspheres containing diltiazem hydrochloride by an aqueous coacervation technique," Pharmaceutica Acta Helvetiae, vol. 73, no. 4, pp. 187192, 1998.

[9] T. Mohima, I. Dewan, S. M. A. Islam, S. Rana, and A. Hossain, "Encapsulation of zidovudine in different cellulosic acrylic and methacrylic polymers loaded microspheres: in vitro characterization and compatibility studies," International Journal of Pharmacy and Pharmaceutical Sciences, vol. 7, no. 1, pp. 486495, 2015.

[10] J. O. Carnali and M. S. Naser, "The use of dilute solution viscometry to characterize the network properties of carbopol microgels," Colloid and Polymer Science, vol. 270, no. 2, pp. 183193, 1992.

[11] G. C. Bazzo, A. T. de Macedo, J. P. Crenca et al., "Microspheres prepared with biodegradable PHBV and PLA polymers as prolonged-release system for ibuprofen: in vitro drug release and in vivo evaluation," Brazilian Journal of Pharmaceutical Sciences, vol. 48, no. 4, pp. 773-780, 2012.

[12] T. Higuchi, "Mechanism of sustained action medication, theoretical analysis of rate of release of solid drugs dispersed in solid matrices," Journal of Pharmaceutical Sciences, vol. 52, pp. 11451149, 1963.

[13] R. W. Korsmeyer, R. Gurny, E. M. Doelker, P. Buri, and N. A. Peppas, "Mechanisms of solute release from porous hydrophilic polymers," International Journal of Pharmaceutics, vol. 15, no. 1, pp. 25-35, 1983.

[14] D. J. Drucker, "Modeling of drug release from sustained release polymers," Europian Journal of Pharmaceutical Technology, vol. 36, pp. 1696-1705, 2006. 

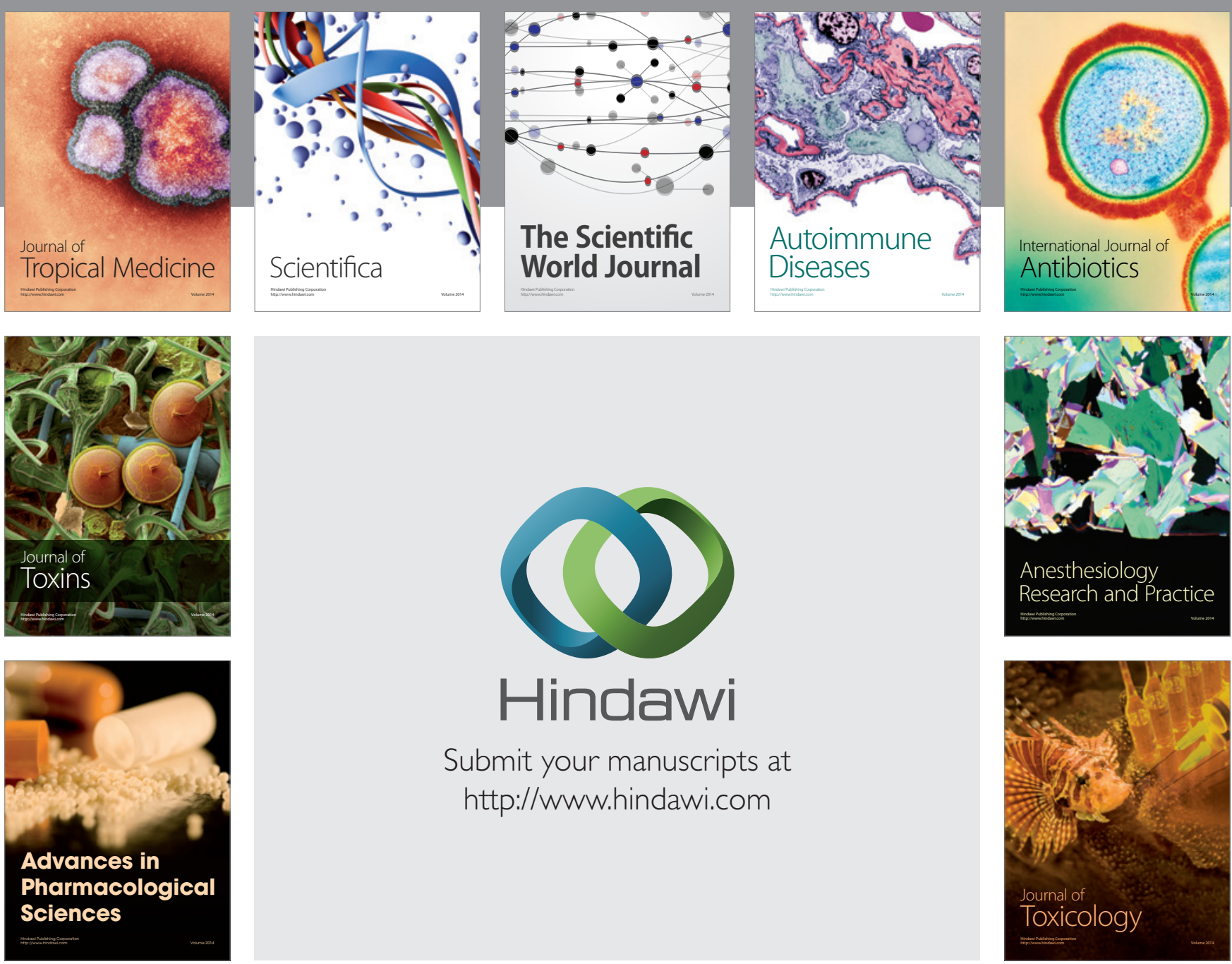

\section{Hindawi}

Submit your manuscripts at

http://www.hindawi.com
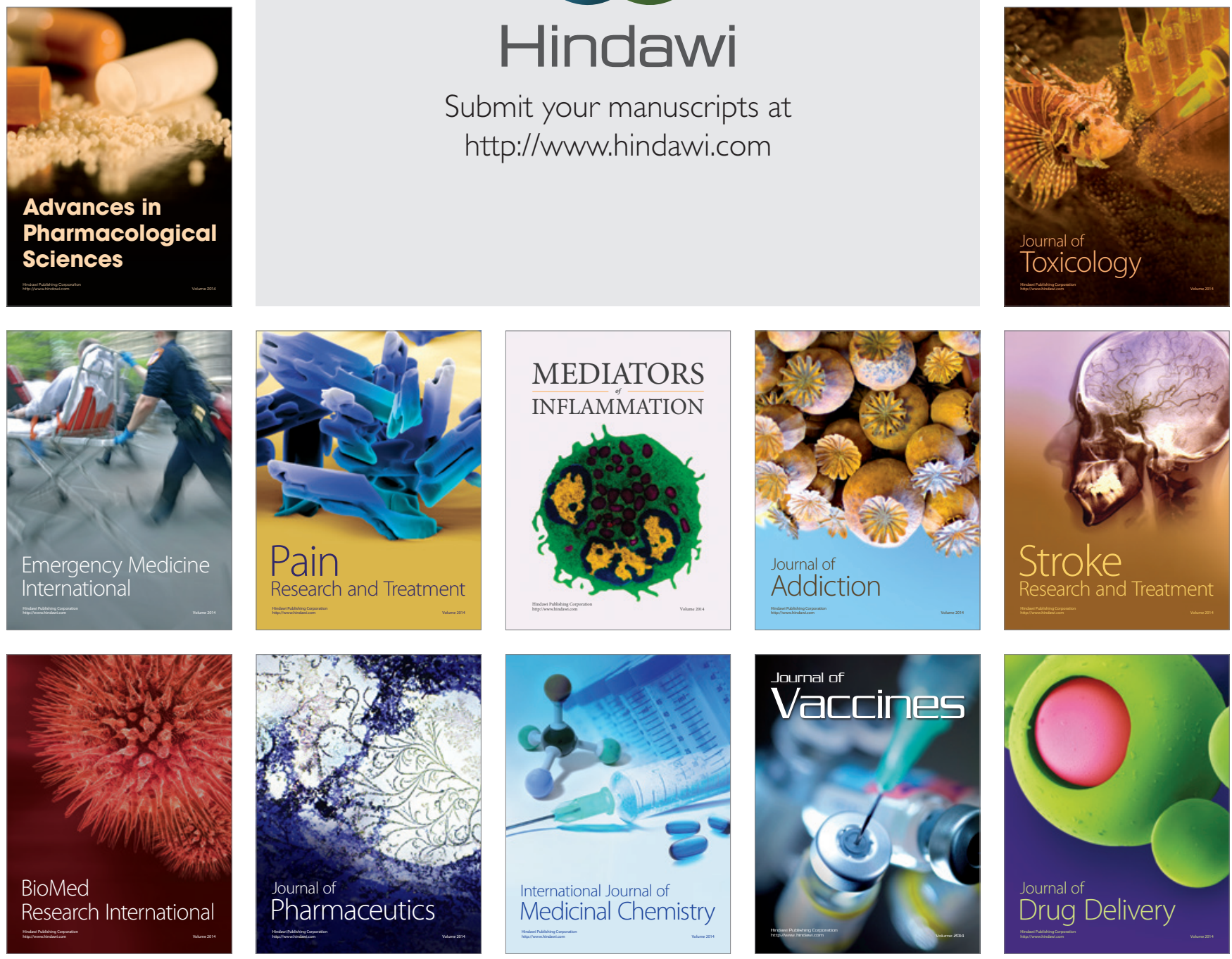